\title{
SOME INVARIANTS OF PRETZEL LINKS
}

\author{
Dongseok Kim and Jaeun LeE
}

\begin{abstract}
We show that nontrivial classical pretzel knots $L(p, q, r)$ are hyperbolic with eight exceptions which are torus knots. We find Conway polynomials of $n$-pretzel links using a new computation tree. As applications, we compute the genera of $n$-pretzel links using these polynomials and find the basket number of pretzel links by showing that the genus and the canonical genus of a pretzel link are the same.
\end{abstract}

\section{INTRODUCTION}

Let $L\left(p_{1}, p_{2}, \ldots, p_{n}\right)$ be an $n$-pretzel link in $\mathbb{S}^{3}$ where $p_{i} \in \mathbb{Z}$ represents the number of half twists as depicted in Figure 1. In particular, if $n=3$, it is called a classical pretzel link, denoted by $L(p, q, r)$. If $n$ is odd, then an $n$-pretzel link $L\left(p_{1}, p_{2}, \ldots, p_{n}\right)$ is a knot if and only if none of two $p_{i}$ 's are even, a pretzel knot is denoted by $K\left(p_{1}, p_{2}, \ldots, p_{n}\right)$. If $n$ is even, then $L\left(p_{1}, p_{2}, \ldots, p_{n}\right)$ is a knot if and only if only one of the $p_{i}$ 's is even. Generally the number of even $p_{i}$ 's is the number of components, unless $p_{i}$ 's are all odd. Since pretzel links have nice structures, they have been studied extensively. For example, several polynomials of pretzel links have been calculated $[13,15,22,28]$. Shinohara calculated the signature of pretzel links [34]. Two and three fold covering spaces branched along pretzel knots have been described $[4,19]$. For notations and definitions, we refer to [2].

A link $L$ is almost alternating if it is not alternating and there is a diagram $D_{L}$ of $L$ such that one crossing change makes the diagram alternating; we call $D_{L}$ an almost alternating diagram. One of the classifications of links is that they are classified by hyperbolic, torus or satellite links [2]. First we show that classical pretzel links are prime and either alternating or almost alternating. Menasco has shown that prime alternating knots are either hyperbolic or torus knots [24]. It has been generalised by Adams that prime almost alternating knots are either hyperbolic or torus knots [1]. It is known that no satellite knot is an almost alternating knot [17]. Thus, we can classify classical pretzel knots completely by hyperbolic or torus knots.

\section{Received 18th October, 2006}

The first author was supported by Korea Research Foundation Grant funded by Korea Government (MOEHRD, Basic Research Promotion Fund) (KRF-2006-351-C00005). The second author was supported in part by $\mathrm{Com}^{2} \mathrm{MaC}-\mathrm{KOSEF}$ (R11-1999-054).

The author would like to thank Cameron Gordon for helpful discussion, valuable comments on this work.

Copyright Clearance Centre, Inc. Serial-fee code: 0004-9727/07 \$A2.00+0.00. 


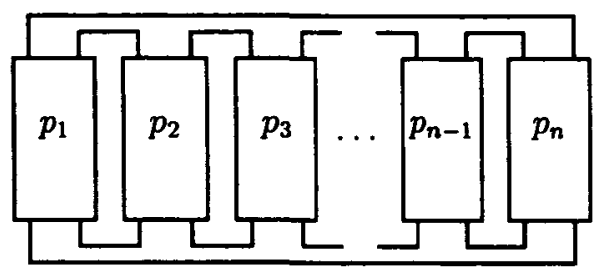

Figure 1: An $n$-pretzel link $L\left(p_{1}, p_{2}, \ldots, p_{n}\right)$

Let $L$ be a link in $\mathbb{S}^{3}$. A compact orientable surface $\mathcal{F}$ is a Seifert surface of $L$ if the boundary of $\mathcal{F}$ is $L$. The existence of such a surface was first proven by Seifert using an algorithm on a diagram of $L$, named after him as Seifert's algorithm [33]. The genus of a link $L$ can be defined by the minimal genus among all Seifert surfaces of $L$, denoted by $g(L)$. A Seifert surface $\mathcal{F}$ of $L$ with the minimal genus $g(L)$ is called a minimal genus Seifert surface of $L$. A Seifert surface of $L$ is canonical if it is obtained from a diagram of $L$ by applying Seifert's algorithm. Then the minimal genus among all canonical Seifert surfaces of $L$ is called the canonical genus of $L$, denoted by $g_{c}(L)$. A Seifert surface $\mathcal{F}$ of $L$ is said to be free if the fundamental group of the complement of $\mathcal{F}$, namely, $\pi_{1}\left(\mathbb{S}^{3}-\mathcal{F}\right)$ is a free group. Then the minimal genus among all free Seifert surfaces of $L$ is called the free genus for $L$, denoted by $g_{f}(L)$. Since any canonical Seifert surface is free, we have the following inequalities,

$$
g(L) \leqslant g_{f}(L) \leqslant g_{c}(L) .
$$

There are many interesting results about the above inequalities $[5,8,21,25,29,32]$. Gabai has geometrically shown that the minimal genus Seifert surface of $n$-pretzel links can be found as a Murasugi sum using Thurston norms and proved that the Seifert surfaces obtained by applying Seifert's algorithm to the standard diagram of $L\left(2 k_{1}\right.$ $\left.+1,2 k_{2}+1, \ldots, 2 k_{n}+1\right)$ and $L\left(2 k_{1}, 2 k_{2}, \ldots, 2 k_{n}\right)$ are minimal genus Seifert surfaces [12]. There is a classical inequality regarding the Alexander polynomial and the genus $g(L)$ of a link $L$ : G. Torres showed the following inequality,

$$
2 g(L) \geqslant \operatorname{degree} \Delta_{L}-\mu+1
$$

where $\Delta_{L}$ is the Alexander polynomial of $L$ and $\mu$ is the number of components of $L$ [36]. Crowell showed that the equality in inequality (1) holds for alternating links [8]. Cimasoni has found a similar inequality from multi-variable Alexander polynomials [6]. In fact, we can find the genera of oriented $n$-pretzel links from the inequality (1) and the Conway polynomial found in section 3 , that is, we shall show that the equality in inequality ( 1 ) holds for all $n$-pretzel links with at least one even crossing. For pretzel links $L\left(2 k_{1}, 2 k_{2}, \ldots, 2 k_{n}\right)$ with all possible orientations, Nakagawa showed that a genus and a canonical genus are the same [28]. The idea of Nakagawa [28] can be extended 
to arbitrary $n$-pretzel links, that is, we can show that these three genera $g(L), g_{f}(L)$ and $g_{c}(L)$ are the same.

Some of Seifert surfaces of links feature extra structures. Seifert surfaces obtained by plumbings annuli have been studied extensively for the fibreness of links and surfaces $[10,11,12,14,26,29,30,35]$. Rudolph has introduced several plumbed Seifert surfaces [31]. Let $A_{n} \subset \mathbb{S}^{3}$ denote an $n$-twisted unknotted annulus. A Seifert surface $\mathcal{F}$ is a basket surface if $\mathcal{F}=D_{2}$ or if $\mathcal{F}=\mathcal{F}_{0} *_{\alpha} A_{n}$ which can be constructed by plumbing $A_{n}$ to a basket surface $\mathcal{F}_{0}$ along a proper arc $\alpha \subset D_{2} \subset \mathcal{F}_{0}$ [31]. A basket number of a link $L$, denoted by $b k(L)$, is the minimal number of annuli used to obtain a basket surface $\mathcal{F}$ such that $\partial \mathcal{F}=L[3,16]$. As a consequence of the results in section 4 and a result [3, Corollary 3.3], we find the basket number of $n$-pretzel links.

The outline of this paper is as follows. In section 2, we mainly focus on the classical pretzel links $L(p, q, r)$. We find Conway polynomial of $n$-pretzel links in section 3. In section 4 , we study the genera of $n$-pretzel links. In section 5 , we compute the basket number of $n$-pretzel links.

\section{Classical pREtzel links $L(p, q, r)$}

2.1. Almost alternating. One can see that $L(p, q, r)$ is alternating if $p, q, r$ have the same signs. Since every alternating link (including any unlink) has an almost alternating diagram, we are going to show that every nontrivial pretzel link has an almost alternating diagram. Since the notation depends on the choice of,+- crossings, it is sufficient to show that $L(-p, q, r)$ has an almost alternating diagram where $p, q, r$ are positive. In particular, one might expect that $L(-1, q, r)$ is almost alternating, but surprisingly it is also alternating.

THEOREM 2.1. For positive integers $p, q$ and $r, L(-1, q, r)$ is an alternating link and $L(-p, q, r)$ has an almost alternating diagram.

Proof: One can see that $L(q,-1, r)$ is isotopic to $L(q-2,1, r-2)$ as shown in Figure 2. For the second part, see Figure 3.

THEOREM 2.2. All nontrivial pretzel knots $K(p, q, r)$ are either torus knots or hyperbolic knots.

PROOF: The key ingredient of theorem is that prime alternating (almost alternating) knots are either hyperbolic or torus knots [24, Corollary 2] ([1, Corollary 2.4], respectively). Since every pretzel knot has an almost alternating diagram by Theorem 2.1 , we need to show that all nontrivial classical pretzel knots are prime. Since no two of $p, q, r$ are even, there are two cases:

(i) all of them are odd,

(ii) exactly one is even. 

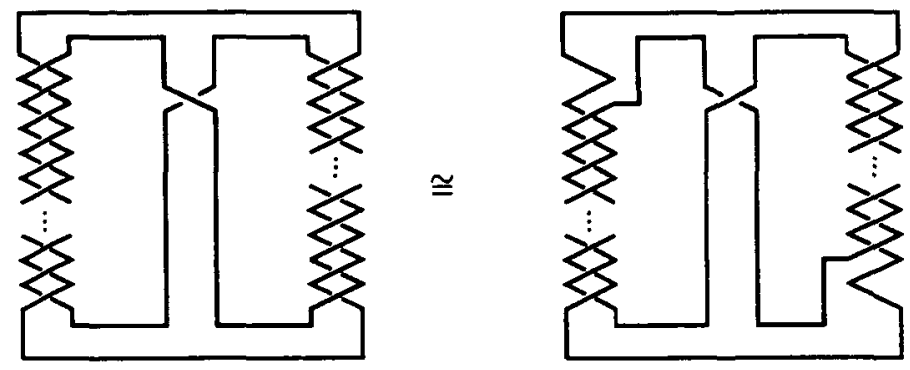

Figure 2: An alternating diagram of $L(q,-1, r)$.
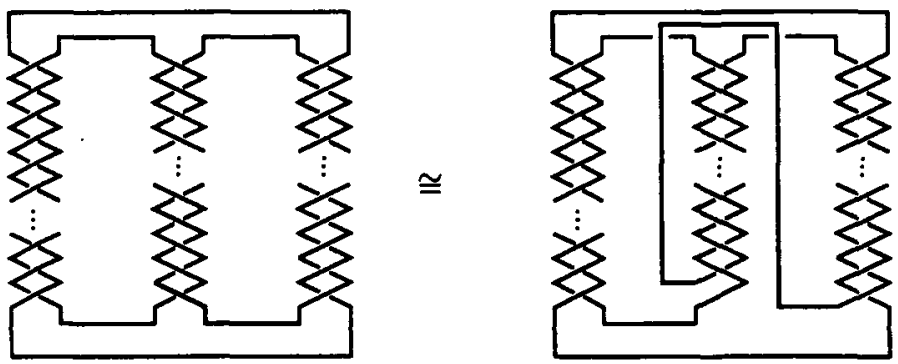

Figure 3: An almost alternating diagram of $L(p,-q, r)$.

(i) $p \equiv q \equiv r \equiv 1(\bmod 2)$. For this case, we can use the genus of $K=K(p, q, r)$. Suppose $K=K_{1} \# K_{2}$. Since a Seifert surface of $K$ is the punctured torus, it has genus 1 as described in the left top of Figure 2. But $1=g(K)=g\left(K_{1}\right)+g\left(K_{2}\right)$. Thus one of $g\left(K_{1}\right)$ or $g\left(K_{2}\right)$ has to be 0 , that is, one of $K_{i}$ is trivial. Therefore $K$ cannot be decomposed as a connected sum of two nontrivial knots.

(ii) Suppose that $p$ is even that is, $p=2 l$, and $q, r$ are odd. Then it is easy to see that the left two twisting parts form a prime tangle (except when $|p|=2 l$ and $|q|=1$ ). The right part is an untangle, but since $r$ is odd, we can use a result of Lickorish [23, Theorem 3] to conclude that $K(2 l, q, r)$ is prime. For the above exceptional cases, we can assume that $|r|=1$ because we can choose $|q| \geqslant|r|$. So all possible cases are $K(2 l, \pm 1, \mp 1), K(2 l, 1,1)$ and $K(2 l,-1,-1)$. But the first one is the unknot and the other two can be deformed to $K(p, q, r)$ of all odd crossings, i.e, $K(2 l,-1,-1)=K(2 l-1,1,1)$ and $K(2 l, 1,1)=K(2 l+1,-1,-1)$. This completes the proof.

2.2. Prime torus PRETZEL KNOTS. The primary goal of this section is to decide which classical pretzel knots are torus knots. For convenience, the $(m, n)$ torus link is denoted by $T_{(m, n)}$. One can see that all 2 -string torus links are alternating. C. Adams has conjectured that only $(3,4)$ and $(3,5)$ torus knots are almost alternating [1]. One can 
see that $K(-2,3,3)$ is the $(3,4)$ torus knot and $K(-2,3,5)$ is the $(3,5)$ torus knot. Since the branched double cover of a torus link is a Seifert fibred space with the base surface $\mathbb{S}^{2}$ and at most three exceptional fibres, and the branched double cover of a nontrivial $n$-pretzel link is a Seifert fibred space with $n$ exceptional fibres, there will be no torus knot of the form $K\left(p_{1}, p_{2}, \cdots, p_{n}\right)$ for $n \geqslant 4$ and $\left|p_{i}\right| \geqslant 2$.

To find all torus knots, we use the Jones polynomials of $K(2 l, q, r)$ because the genera of pretzel knots tell us that no $K(p, q, r)$, with $p, q, r$ all odd, is a torus knot except the unknot and trefoil, and it is known that $K(p,-1,1)$ is the unknot and $K( \pm 1, \pm 1, \pm 1)$ are trefoils, which are the only torus knots of genus 1 . Remark that the genus of an $(m, n)$ torus knot is $(m-1)(n-1) / 2$. The Jones polynomial of an $(m, n)$ torus link $(m \leqslant n)$ is given by equation (2) if $m$ is odd, by equation (3) if $4 \leqslant m$ is even, and by equation (4) if $m=2$ and $n$ is even. This is due to the original work by Jones [18] but still there is no combinatorial proof for these formulae.

$$
\begin{aligned}
& -t^{(m-1)(n-1) / 2}\left[t^{m+n-2}+t^{m+n-4}+\cdots+t^{n+1}-t^{m-1}-\cdots-t^{2}-1\right], \\
& -t^{(m-1)(n-1) / 2}\left[t^{m+n-2}+t^{m+n-4}+\cdots+t^{n}-t^{n-1}-\cdots-t^{2}-1\right], \\
& -t^{(n-1) / 2}\left[t^{n}-t^{n-1}+t^{n-2}-\cdots-t^{3}+t^{2}+1\right] .
\end{aligned}
$$

Using a formula for the Jones polynomials of $n$-pretzel knots in [22], we find the following lemma. Since the Jones polynomial of the mirror image $\bar{L}$ of $L$ can be found by $V_{\bar{L}}(t)=V_{L}\left(t^{-1}\right)$, we may assume $q, r$ are positive integers.

LEMMA 2.3. Let $l, q, r$ be positive integers. Let $k=2 l+q+r$.

$$
\begin{array}{rlrl}
V_{K(2,1, r)} & =t^{(r+1) / 2-(2+1)}\left(t^{r+2+1}-2 t^{r+2}+2 t^{r+1}-\cdots+2 t^{3}-t^{2}+t-1\right), \\
V_{K(2 l, q, r)} & =t^{(q+r) / 2-(2 l+1)}\left(t^{k}-2 t^{k-1}+3 t^{k-2}-4 t^{k-3}+\cdots-3 t^{2}+t-1\right), \text { if } l \geqslant 1, \\
V_{K(2 l,-q, r)} & =-t^{(-4 l-3 q+r) / 2}\left(t^{q+r}-t^{q+r-1}+\cdots-t+1\right) \quad \text { if } q>1, \\
V_{K(-2,1, r)} & =-t^{(r+1) / 2}\left(t^{r+2}-t^{r+1}+t^{r}-\cdots+t^{3}-t^{2}-1\right), & \\
V_{K(-2,3,3)} & =-t^{3}\left(t^{5}-t^{2}-1\right), & \\
V_{K(-2,3,5)} & =-t^{4}\left(t^{6}-t^{2}-1\right), & \\
V_{K(-2,3, r)} & =-t^{(3+r) / 2}\left(t^{3+r-2}-t^{r}+\cdots-t^{2}-1\right) & \text { if } r \geqslant 7, \\
V_{K(-2, q, r)} & =-t^{(q+r) / 2}\left(-t^{q+r-1}+2 t^{q+r-2}-\cdots-t^{2}-1\right) & & \text { if } q, r \geqslant 5, \\
V_{K(-2 l, q, r)} & =-t^{(q+r) / 2}\left(a t^{*}+\cdots \pm t \mp 1\right) & & \text { if } l, q, r>1 .
\end{array}
$$

By comparing Jones polynomials of pretzel knots in Lemma 2.3 and Jones polynomials of torus knots in equation (2), (3) and (4), we find the following theorem.

THEOREM 2.4. The following are the only nontrivial pretzel knots which are torus knots. 
(1) $K(p, \pm 1, \mp 1)$ are unknots for all $p$.

(2) $K( \pm 1, \pm 1, \pm 1)$ are $(2, \pm 3)$ torus knots.

(3) $K( \pm 2, \mp 1, \pm r)$ are $(2, \pm r \mp 2)$ torus knots.

(4) $K(\mp 2, \pm 3, \pm 3), K(\mp 2, \pm 3, \pm 5)$ are $(3, \pm 4),(3, \pm 5)$ torus knots, respectively.

Proof: We only need to consider $K(2 l, q, r)$. We can see that $K(2,-1, r)$ can be deformed to $K(0,1, r-2)$ by a move shown in Figure 2. The coefficient of $t^{1}$ and the second leading coefficient of the Jones polynomial of a torus knot are zero, but by Lemma 2.3 these are possible only for $K(-2,3,3), K(-2,3,5)$ and their mirror images. But the number of terms in the Jones polynomials of these knots is 3 , and only $(3, n)$ torus knots have this property. By comparing the terms of the highest degree, we conclude that $K(\mp 2, \pm 3, \pm 3)$ and $K(\mp 2, \pm 3, \pm 5)$ are the remaining non-alternating torus knots.

2.3. Minimal genus Seifert surfaces. When one applies Seifert's algorithm to a diagram of a link $L$, in general one may not get a minimal genus Seifert surface. In fact, Moriah found infinitely many knots which have no diagram on which Seifert's algorithm produces a minimal genus surface [25]. But it is known that a minimal genus Seifert surface can be obtained from an alternating diagram by applying Seifert's algorithm [27] and more generally, alternative links [20]. We prove that the Seifert surface obtained by applying Seifert's algorithm to the diagram in Figure 4 of a pretzel knot $K(p, q, r)$ is a minimal genus Seifert surface. Since $K(2 l, q, r)$ and its mirror image are alternating, without loss of a generality, we only need to find Alexander polynomials of $K(-2 l, q, r)$ and $K(-2 l, q,-r)$.

LемMA 2.5. Let $l, q, r$ be positive integers.

$$
\begin{aligned}
\Delta_{K(-2 l, q, r)}(t) & =t^{-(q+r) / 2}\left(l t^{q+r}-(2 l-1) t^{q+r-1}+\cdots-(2 l-1) t+l\right), \\
\Delta_{K(-2 l, q,-r)}(t) & =t^{-(q+r-2) / 2}\left(t^{q+r-2}-2 t^{q+r-3}+\cdots-2 t+1\right) .
\end{aligned}
$$

Proof: One can prove inductively the lemma by the following recurrence formulae which come from the skein relations, and the formulae for the Alexander polynomial of the $(2, p)$ torus links.

$$
\begin{aligned}
\Delta_{T_{(2, \pm p)}}(t) & =t^{(1-p) / 2}\left(t^{p-1}-t^{p-2}+\cdots-t+1\right) \text { if } p \text { is odd, } \\
\Delta_{T_{(2, \pm p)}}(t) & =t^{(1-p) / 2}\left(-t^{p-1}+t^{p-2}+\cdots-t+1\right) \text { if } p \text { is even, } \\
\Delta_{K(-2, q, \pm r)}(t) & =\Delta_{T_{(2, q)}}(t) \Delta_{T_{(2, r)}}(t)+\left(t^{-1 / 2}-t^{1 / 2}\right) \Delta_{T_{(2, q \pm r)}}(t) \\
\Delta_{K(-2 l, q, \pm r)}(t) & =\Delta_{K(-2(l-1), q, \pm r)}(t)+\left(t^{-1 / 2}-t^{1 / 2}\right) \Delta_{T_{(2, q \pm r)}}(t) .
\end{aligned}
$$



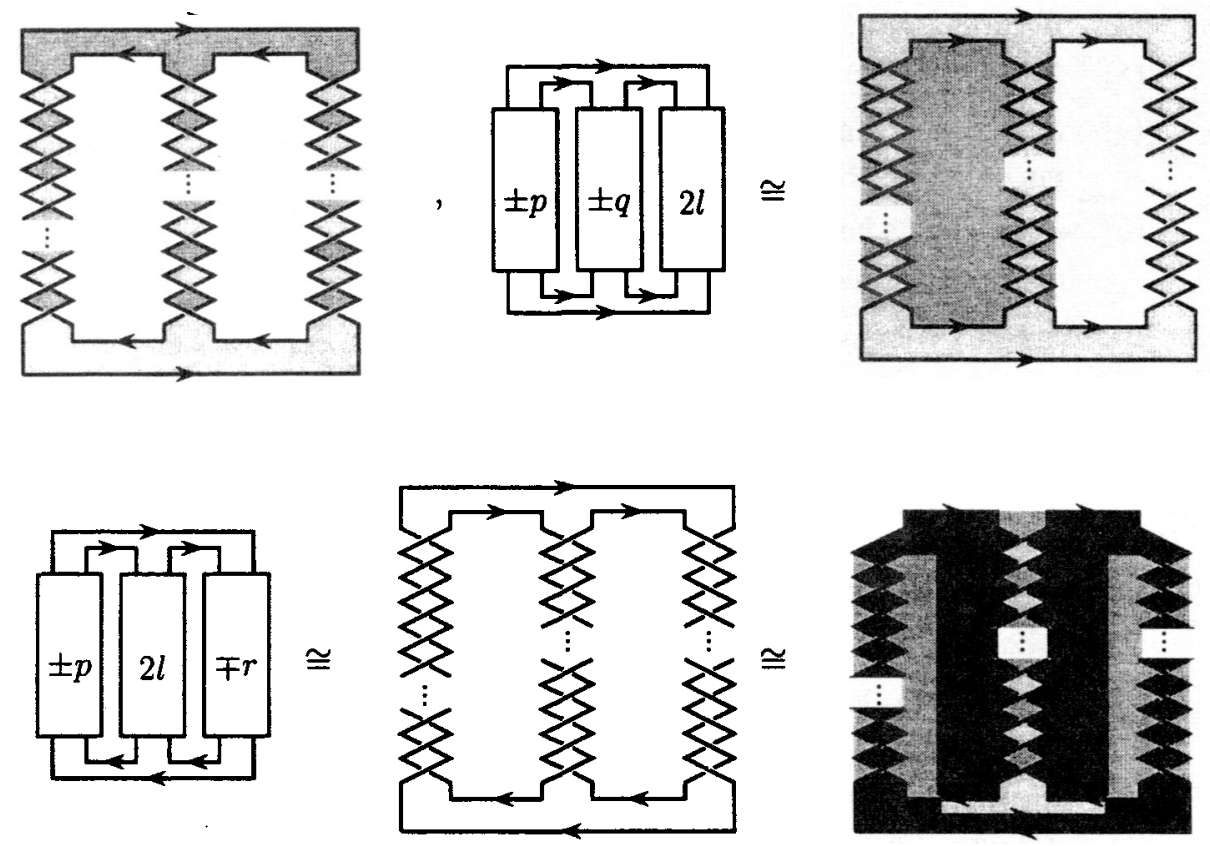

Figure 4: Minimal genus Seifert surfaces of the pretzel knots $K(p, q, r)$.

THEOREM 2.6. The surface obtained by applying Seifert's algorithm to the pretzel knot $K(p, q, r)$ as in Figure 4 is a minimal genus Seifert surface, if $1 /|p|+1 /|q|+1 /|r|$ $\leqslant 1$.

Proof: We consider two cases:

(i) all of $p, q, r$ are odd,

(ii) exactly one of $p, q, r$ is even.

For the first case, the first Seifert surface in Figure 4 is clearly a minimal genus since its genus is 1 unless $K(p, q, r)$ is the unknot. But it can not be the unknot by the hypothesis. For the second case, we can consider $K(-2 l, q, \pm r), K(-2 l, q, \pm r)$ or their mirror images, where $l, q, r$ are positive. Their canonical Seifert surfaces are given in Figure 4 . To prove these surfaces are minimal genus Seifert surfaces, first we find $2 g(K(-2 l, q, \pm r))$ $\geqslant q+r-1 \pm 1$ using the Alexander polynomials of $K(-2 l, q, r)$ and $K(-2 l, q,-r)$ given in Lemma 2.5 and inequality (1). But the genus of the second Seifert surface in Figure 4 is $(q+r) / 2$, and the third surface in Figure 4 is $(q+r-2) / 2$. It completes the proof. $\square$

By combining Theorem 2.4 and Theorem 2.6, we find the following corollary.

COROLlary 2.7. The genus of $K(p, q, r)$ is as follows. 
(1) $K(p, \pm 1, \mp 1), K( \pm 2, \mp 1, \pm 3)$ have genus 0 for all $p$.

(2) $K(p, q, r)$ has genus 1 if $p \equiv q \equiv r \equiv 1(\bmod 2)$ and we are not in case 1$)$.

(3) $K( \pm 2, \mp 1, \pm r)$ has genus $(|r-2|-1) / 2$.

(4) $K(\mp 2 l, q, r)$ has genus $(|q|+|r|) / 2$ if $q, r$ have the same sign and we are not in any of the previous cases.

(5) $K(\mp 2 l, q, r)$ has genus $(|q|+|r|-2) / 2$ if $q, r$ have different signs and we are not in cases (1), (2) or (3).

For classical pretzel links, one can see that $L\left(2 l_{1}, 2 l_{2}, 2 l_{3}\right)$ has genus 0 . For $L\left(2 l_{1}, 2 l_{2}\right.$, $r$ ), we are going to see more interesting results for the genus because there is a freedom to choose orientations of the components. But, Lemma 2.5 remains true for arbitrary integers $q, r$, so we can find the following corollary.

Corollary 2.8. The genus of the link $L\left(2 l_{1}, 2 l_{2}, r\right)$, where $\left|l_{1}\right| \geqslant\left|l_{2}\right|, l_{1}, l_{2}$ $>0$ (unless we indicate differently) and $r \geqslant 0$, is as follows.

(1) $L\left(2 l_{1}, 2 l_{2}, \pm r\right)$ has genus 0 if $r \equiv 0(\bmod 2)$ and $l_{1}, l_{2}$ are nonzero integers.

(2) $L\left( \pm 2, \pm 2 l_{2}, \mp 1\right)$ has genus $\left(\left|2 l_{2}-2\right|-2\right) / 2$.

(3) $K\left(\mp 2 l_{1}, \mp 2 l_{2}, \mp r\right)$ has genus $\left(\left|l_{2}\right|+|r|-1\right) / 2$ if we are not in one of the previous cases.

(4) $K\left(\mp 2 l_{1}, \mp 2 l_{2}, \pm r\right)$ has genus $\left(\left|l_{2}\right|+|r|-3\right) / 2$ if we are not in any of the previous cases.

(5) $K\left(\mp 2 l_{1}, \pm 2 l_{2}, \mp r\right)$ has genus $\left(\left|l_{2}\right|+|r|-3\right) / 2$ if we are not in case 1$)$.

(6) $K\left(\mp 2 l_{1}, \pm 2 l_{2}, \pm r\right)$ has genus $\left(\left|l_{2}\right|+|r|-1\right) / 2$ if we are not in case 1$)$ and $\left|l_{1}\right|>\left|l_{2}\right|$, or has genus $\left(\left|l_{2}\right|+|r|-3\right) / 2$ if we are not in case 1$)$ and $\left|l_{1}\right|=\left|l_{2}\right|$.

PROOF: We follow the proof of Theorem 2.4 and Theorem 2.6 carefully; if $r= \pm 1$, the link will have two representatives by the move we used in the proof of Theorem 2.1, we get the result, with a note that we have a freedom to choose an orientation of the component which goes through two even crossing boxes.

\section{CONWAY POLYNOMIALS OF $n$-PRETZEL LINKS}

To find the polynomial invariants of $n$-pretzel links, we shall use a computation tree: a computation tree of a link polynomial $P_{L}$ is an edge weighted, rooted binary tree whose vertices are links, the root of the tree is $L$, two vertices $L_{1}, L_{2}$ are children of a vertex $L_{p}$ if

$$
P_{L_{p}}=w\left(L_{p(1)}\right) P_{L_{1}}+w\left(L_{p(2)}\right) P_{L_{2}}
$$

and $w\left(L_{p(i)}\right)$ is the weight on the edge between $L_{p}$ and $L_{i}$. One can see that the link polynomial $P_{L}$ can be computed as follows,

$$
P_{L}=\sum_{L_{v} \in \mathcal{C}} \prod_{L_{p} \in \mathcal{P}\left(L_{v}\right)} w\left(L_{p(i)}\right) P_{L_{v}},
$$




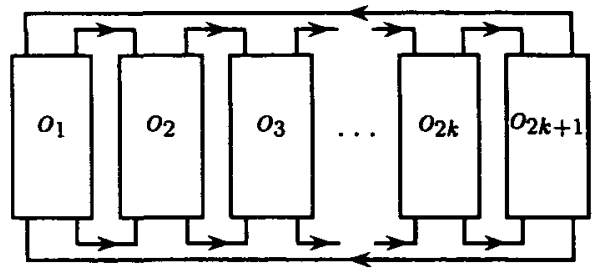

(5)

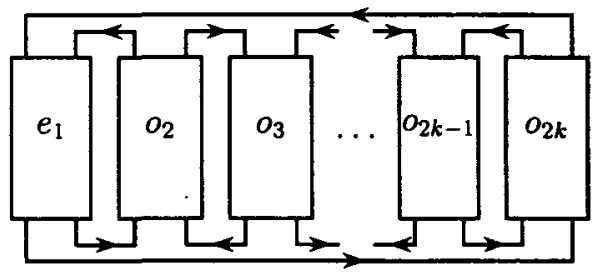

(7)

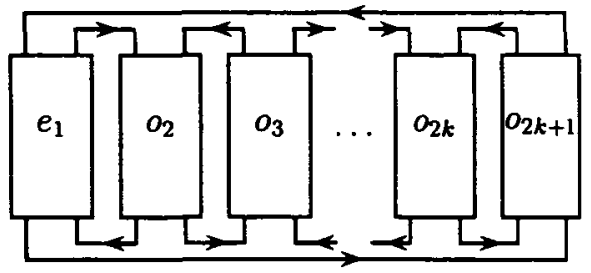

(6)

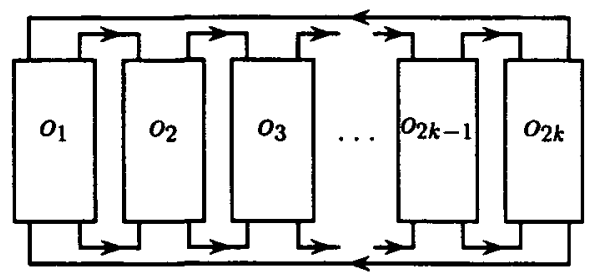

$(8,9)$

Figure 5: all oriented $n$-pretzel knots $L\left(p_{1}, p_{2}, \ldots, p_{n}\right)$

where $\mathcal{L}$ is the set of all vertices of valence 1 and $\mathcal{P}\left(L_{v}\right)$ is the set of all vertices of the path from the root to the vertex $L_{v}$. In general, it is easy to find $P_{L}$ if we repeatedly use the skein relations until each vertex $L_{v}$ becomes an unlink. Moreover, one can replace links by other for a convenience of the computation. For instance, Franks and Williams used braids to find a beautiful result on Jones polynomial [9].

To compute Conway Polynomials of $n$-pretzel links, we shall use a new notation for $n$-pretzel links which will be used for vertices of a computation tree. We called a rectangle in Figure $5 a$ box and the link moves in the same direction in a box if it has the orientation as in the second box from the left of the diagram (6) of Figure 5, in the opposite directions if it has the orientation as in the first box from the left of the diagram (6) in Figure 5. If we have a box for which two strings move in the opposite directions and we use the skein relation at this box, then the resulting links have either less number of the boxes or less number of crossings. One can see that an opposite direction can be happened only for a box with even number of crossings (but this is not sufficient) except in the case that $n$ is even and all the $p_{i}$ 's are odd (we shall handle this case separately). Suppose we have at least one even crossing box. We may assume that it is $p_{1}=2 l_{1}$. Let us remark that the Conway polynomial vanishes for split links. The following is our new notation for $n$-pretzel links. From a given $n$-pretzel link $L$ with an orientation $O$, we can represent $L$ by a vector in $\left(\mathbb{Z} \times \mathbb{Z}_{2}\right)^{n}$ such as $\left(p_{1}^{\varepsilon_{1}}, p_{2}^{\varepsilon_{2}}, \ldots, p_{n}^{\varepsilon_{n}}\right)$, where $\varepsilon_{i}=1(-1)$ if the link moves in the same(opposite, respectively) direction in the box corresponding to $p_{i}$ with respect to the given orientation $O$. Write $p_{i}^{1}=p_{i}$. First we find the following 
recursive formula,

$$
\begin{aligned}
\nabla_{L\left(p_{1}^{\varepsilon_{1}}, p_{2}^{\varepsilon_{2}}, \ldots, p_{i}^{-1}, \ldots, p_{n}^{\varepsilon_{n}}\right)} & =\nabla_{T_{\left(2, p_{1}^{\varepsilon_{1}}\right)}} \nabla_{T_{\left(2, p_{2}^{\varepsilon_{1}}\right)}} \ldots \widehat{\nabla_{T_{\left(2, p_{i}^{-1}\right.}}} \ldots \nabla_{T_{\left(2, p_{n}^{\varepsilon_{n}}\right)}} \\
& -l_{i} z \nabla_{L\left(p_{1}^{\varepsilon_{1}}, p_{2}^{\varepsilon_{2}}, \ldots, \widehat{p_{i}^{-1}}, \ldots, p_{n}^{\varepsilon_{n}}\right)}
\end{aligned}
$$

where the term under ${ }^{-}$is deleted.

By repeatedly using above formulae, we can make a computation tree such that there is no negative $\varepsilon_{i}$ for the representative at each vertex of valence 1 . Then, we can expand $\left(\ldots, p_{i}, \ldots\right)$ into $\left(\ldots, p_{i} \pm 1\left(=p_{i}^{\prime}\right), \ldots\right)$ and $\left(\ldots, p_{i} \pm 2, \ldots\right)$ with suitable weights on edges, 1 or $\pm z$ where $\left|p_{i}\right|>\left|p_{i}^{\prime}\right|$. We can keep on expanding at the crossings until all the entries in the vectors of vertices of valence 1 are either 0 or \pm 1 . At this stage, if it has more than two 0's then we stop the expansion and change the vertex to zero because it is a split link. If it has only one zero, it is a composite link of $T_{\left(2, p_{i}\right)}$ 's. Otherwise, we change the vector to an integral value $m$, the sum of the signs of entries in the vector. In fact, it is the closed braid of two strings represented by $\sigma_{1}^{m}$. Therefore, we can compute the Conway polynomial of a link $L$ using this computation tree and the Conway polynomial of closed 2-braids.

3.1. CONWAY POLYNOMIAL OF $n$-PRETZEL KNOTS. The general figures of $n$-pretzel knots are given in Figure 5 (the right-top one is a two components link) where $e_{1}$ $=2 l, o_{i}=2 k_{i}+1$. We can see that there is at most one box in which the knot moves in opposite directions. But for a two component link, all boxes might move in opposite directions for the orientation which is not in Figure 5. Counterclockwise from the topright, we get representatives, $\left(o_{1}^{-1}, o_{2}^{-1}, \ldots, o_{2 k}^{-1}\right),\left(o_{1}, o_{2}, \ldots, o_{2 k}\right),\left(o_{1}^{-1}, o_{2}^{-1}, \ldots, o_{2 k+1}^{-1}\right)$, $\left(e_{1}^{-1}, o_{2}, o_{3}, \ldots, o_{2 k+1}\right)$ and $\left(e_{1}, o_{2}, o_{3}, \ldots, o_{2 k}\right)$. By using a computation tree for these representatives, we find Theorem 3.1. For convenience, we abbreviate $\nabla_{T_{(2, n)}}$ by $\nabla_{n}$ throughout the section.

THEOREM 3.1. Let $e_{1}^{\prime}=\operatorname{sign}\left(e_{1}\right)\left(\left|e_{1}\right|-1\right), o_{i}^{\prime}=\operatorname{sign}\left(o_{i}\right)\left(\left|o_{i}\right|-1\right), \alpha=\sum_{i=2}^{n} \operatorname{sign}\left(o_{i}\right)$ and $\beta=\operatorname{sign}\left(e_{1}\right)$. The Conway polynomials of $n$-pretzel knots in Figure 5 are

$$
\begin{aligned}
& \nabla_{L\left(o_{1}, o_{2}, o_{3}, \ldots, o_{n}\right)}=\sum_{i=0}^{(n-1) / 2} a_{i} z^{2 i}, \\
& \nabla_{L\left(e_{1}, o_{2}, o_{3}, \ldots, o_{n}\right)}=\nabla_{o_{2}} \nabla_{o_{3}} \ldots \nabla_{o_{n}}\left[1-l z\left[-\frac{\alpha}{2} z+\sum_{i=2}^{n} \frac{\nabla_{o_{i}^{\prime}}}{\nabla_{o_{i}}}\right]\right] \\
& \nabla_{L\left(e_{1}, o_{2}, o_{3}, \ldots, o_{n}\right)}=\nabla_{o_{2}} \nabla_{o_{3}} \ldots \nabla_{o_{n}}\left[\nabla_{e_{1}^{\prime}}+\nabla_{e_{1}}\left[-\frac{\beta+\alpha}{2} z+\sum_{i=2}^{n} \frac{\nabla_{o_{i}^{\prime}}}{\nabla_{o_{i}}}\right]\right], \\
& \nabla_{L\left(o_{1}, o_{2}, o_{3}, \ldots, o_{n}\right)}=\sum_{i=1}^{(n+1) / 2} a_{i} z^{2 i-1}
\end{aligned}
$$




$$
\nabla_{L\left(o_{1}, o_{2}, o_{3}, \ldots, o_{n}\right)}=\nabla_{o_{1}} \nabla_{o_{2}} \ldots \nabla_{o_{n}}\left[\nabla_{\sum_{i=1}^{n} \operatorname{sign}\left(o_{i}\right)}+\sum_{i=1}^{n} \frac{\nabla_{o_{i}}}{\nabla_{o_{i}}}\right]
$$

where for $L\left(o_{1}, o_{2}, o_{3}, \ldots, o_{n}\right)$ we have two possible orientations because it is a two components link, so we get (8) for $\left(o_{1}^{-1}, o_{2}^{-1}, \ldots, o_{2 k}^{-1}\right)$ and (9) for $\left(o_{1}, o_{2}, \ldots, o_{2 k}\right)$.

Proof: We shall only prove (6) but one can prove the other by a similar argument. In the computation tree, we use skein relation at crossings until vertices of valence 1 in the computation tree up to this point will be $\left(c_{1}, c_{2}, \ldots, c_{n}\right)$ where $c_{i}$ is either 0 or \pm 1 . Since the Conway polynomials of split links vanish, we may assume there are no than one 0 's. The first term in the parenthesis comes from the case where all $\left|c_{i}\right|$ are 1 because it is again the $(2, \alpha)$ torus link horizontally. It is a two component link with linking number $-\alpha / 2$, so its Conway polynomial is $-(\alpha / 2) z$. For the case where only one $c_{i}=0$, the values on edges to the vertex will contribute exactly $\nabla_{o_{i}^{\prime}}$ and the vertex is the composite link of $\left(2, o_{j}\right)$ torus knots $j=2, \ldots, n$ except $i$.

3.2. ConWAY POLYNOMIALS of $n$-PRETZEL Links. Since we have already handled links of all odd crossings, we assume that $n$-pretzel links have at least one even crossing box. Let $L\left(p_{1}, p_{2}, \ldots, p_{n}\right)$ be an $n$-pretzel link and let $s$ be the number of even $p_{i}$ 's. Then it is a link of $s$ components. The Conway polynomial $\nabla_{L}$ depends on the choice of the orientation of $L$. There are $2^{s-1}$ possible orientations of $L$. But one can easily see that the link always moves in the same direction in all boxes of odd crossings for arbitrary orientation. For further purpose, we shall calculate the Conway polynomial of the pretzel link with the following orientations. For the existence of such orientations, we shall prove it in Lemma 4.2: if $n-s$ is even, then there exists an orientation $O$ of $L$ such that the link $L$ moves in the opposite directions in all boxes of even $p_{i}$. If $n-s$ is odd, then there exists an orientation $O$ of $L$ such that the link $L$ moves in the opposite directions in all boxes of even $p_{i}$ except one $p_{t}$ but without loss of a generality we assume that $p_{1}=p_{t}$.

THEOREM 3.2. Let $L\left(p_{1}, p_{2}, \ldots, p_{n}\right)$ be a pretzel link with the above orientation $O$. Let $p_{e_{i}}=2 l_{i}$ be all even and $p_{o_{j}}=2 k_{j}+1$ be all odd. Let $s$ be the number of even $p_{i}$ 's and let $\alpha=\sum_{i=1}^{n-s} \operatorname{sign}\left(p_{o_{i}}\right)$ and $\beta=\operatorname{sign}\left(p_{1}\right)$. Let $p_{i}^{\prime}=\operatorname{sign}\left(p_{i}\right)\left(\left|p_{i}\right|-1\right)$. If $n-s$ is even, then the Conway polynomial of $L\left(p_{1}, p_{2}, \ldots, p_{n}\right)$ is

$$
\left[\prod_{i=1}^{s}\left(-l_{i}\right)\right] z^{s}\left(\prod_{i=1}^{n-s} \nabla_{p_{o_{i}}}\right)\left[-\frac{\alpha}{2} z+\sum_{i=1}^{n-s} \frac{\nabla_{p_{o_{i}}}}{\nabla_{p_{o_{i}}}}\right]+\left[\sum_{i=1}^{s} \prod_{j=1, j \neq i}^{s}\left(-l_{j}\right)\right] z^{s-1} \text {. }
$$

If $n-s$ is odd, then the Conway polynomial of $L\left(p_{1}, p_{2}, \ldots, p_{n}\right)$ is

$$
\left[\prod_{i=2}^{s}\left(-l_{i}\right)\right] z^{s-1}\left(\prod_{i=1}^{n-s} \nabla_{p_{o_{i}}}\right) \nabla_{p_{1}}\left[-\frac{\alpha+\beta}{2} z+\frac{\nabla_{p_{1}^{\prime}}}{\nabla_{p_{1}}}+\sum_{i=1}^{n-s} \frac{\nabla_{p_{o_{i}}}}{\nabla_{p_{o_{i}}}}\right]+\left[\sum_{i=2}^{s} \prod_{j=2, j \neq i}^{s}\left(-l_{j}\right)\right] z^{s-2}
$$


Proof: It is clear by choosing $\left(p_{e_{1}}^{-1}, p_{e_{2}}^{-1}, \ldots, p_{e_{1}}^{-1}, p_{o_{1}}, \ldots, p_{o_{n-s}}\right)$ and $\left(p_{e_{1}}, p_{e_{2}}^{-1}, \ldots\right.$, $\left.p_{e_{s}}^{-1}, p_{o_{1}}, \ldots, p_{o_{n-1}}\right)$, respectively.

More generally, we get the following results by taking $\left(p_{e_{1}}^{-1}, p_{e_{2}}^{-1}, \ldots, p_{e_{t}}^{-1}, p_{e_{t+1}}, \ldots\right.$, $\left.p_{e_{s}}, p_{o_{1}}, \ldots, p_{o_{n-s}}\right)$ for a representative of $L\left(p_{1}, p_{2}, \ldots, p_{n}\right)$ induced by an orientation $O$.

THEOREM 3.3. Let $p_{e_{i}}=2 l_{i}$ be all even and $p_{o_{j}}=2 k_{j}+1$ be all odd. Let $s$ be the number of even $p_{i}$. Let $t$ be the number of even $p_{i}$ in the corresponding boxes in which the link moves in the opposite direction, say $p_{e_{i}}$ where $i=1,2, \ldots, t$. and let $\alpha=\sum_{j=1}^{n-s} \operatorname{sign}\left(p_{o_{j}}\right)$ and $\beta=\sum_{i=t+1}^{s} \operatorname{sign}\left(p_{e_{i}}\right)$. Let $p_{i}^{\prime}=\operatorname{sign}\left(p_{i}\right)\left(\left|p_{i}\right|-1\right)$. Then the Conway polynomial of $L\left(p_{1}, p_{2}, \ldots, p_{n}\right)$ with the orientation $O$ is

$$
\begin{aligned}
{\left[\prod_{i=1}^{t}\left(-l_{i}\right)\right] z^{t}\left(\prod_{i=1}^{n-s} \nabla_{p_{o_{i}}}\right)\left(\prod_{j=1}^{t} \nabla_{p_{e_{j}}}\right)\left[-\frac{\alpha+\beta}{2} z\right.} & +\sum_{i=t+1}^{s} \frac{\nabla_{p_{e_{i}}^{\prime}}}{\nabla_{p_{e_{i}}}} \\
& \left.+\sum_{j=1}^{n-s} \frac{\nabla_{p_{o_{j}}}}{\nabla_{p_{o_{j}}}}\right]+\left[\sum_{i=1}^{t} \prod_{j=1, j \neq i}^{t}\left(-l_{j}\right)\right] z^{t-1} .
\end{aligned}
$$

\section{Genera of $n$-PRETZEL LINKS}

We shall consider the genus of an $n$-pretzel link with at least one even crossing box. Let $F_{L}$ be a Seifert surface of an $n$-pretzel link $L$. For the rest of the section, let $\chi\left(\mathcal{F}_{L}\right)$ be the Euler characteristic of $\mathcal{F}_{L}, V$ be the number of Seifert circles, $E$ be the number of crossings and $F$ be the number of the components of $L$.

4.1. Genera of $n$-PRETzel KNOTS With ONE EVEN $p_{i}$. We divide into two cases:

(i) $n$ is odd,

(ii) $n$ is even.

For the first case: $n$ is odd, we can see that the degree of $\nabla_{K\left(e_{1}, o_{1}, o_{2}, \ldots, o_{n}\right)}$ is

$$
2+\prod_{i=2}^{n} \operatorname{degree}\left(\nabla_{o_{i}}\right)=2+\sum_{i=2}^{n}\left(\left|o_{i}\right|-1\right)
$$

and the coefficient of this leading term is $-l \alpha / 2$ from Theorem 3.1.

Suppose $\alpha$ is nonzero. Then the Seifert surface $\mathcal{F}$ obtained by applying Seifert's algorithm to the diagram in Figure 5 is a minimal genus surface. The genus of the Seifert surface $\mathcal{F}_{K}$ is

$$
\begin{aligned}
g\left(\mathcal{F}_{K}\right) & =\frac{1}{2}\left[2-\chi\left(\mathcal{F}_{K}\right)\right]=\frac{1}{2}(2-V+E-F) \\
& =\frac{1}{2}\left[2-\left(\left|e_{1}\right|+n-2\right)+\left(\left|e_{1}\right|+\sum_{i=2}^{n}\left|o_{i}\right|\right)-1\right]=\frac{1}{2}\left[2+\sum_{i=2}^{n}\left(\left|o_{1}\right|-1\right)\right] \\
& =\frac{1}{2} \text { degree } \nabla_{K\left(e_{1}, o_{1}, o_{2}, \ldots, o_{n}\right)} .
\end{aligned}
$$


Suppose $\alpha=0$. This means that we have the same number of positive and negative twists on odd twists. If we look at the Conway polynomial in equation 6 , we drop exactly one in degree with new leading coefficient 1. It is sufficient to show that the degree of the following term is negative. Remark that $\nabla_{o_{i}}=\nabla_{-o_{i}}$.

$$
\begin{aligned}
-l z\left[-\frac{\alpha}{2} z+\sum_{i=2}^{n} \frac{\nabla_{o_{i}^{\prime}}}{\nabla_{o_{i}}}\right] & =-l\left[0+\sum_{i=2}^{n} \frac{z \nabla_{o_{i}}}{\nabla_{o_{i}}}\right]=-l\left[\sum_{i=2}^{n} \frac{\operatorname{sign}\left(o_{i}\right)\left(\nabla_{\left|o_{i}\right|}-\nabla_{\left|o_{i}\right|-2}\right)}{\nabla_{\left|o_{i}\right|}}\right] \\
& =-l\left[\sum_{i=2}^{n}\left(\operatorname{sign}\left(o_{i}\right)+\frac{\nabla_{\left|o_{i}\right|-2}}{\nabla_{\left|o_{i}\right|}}\right)\right]=-l\left[\sum_{i=2}^{n} \frac{\nabla_{\left|o_{i}\right|-2}}{\nabla_{\left|o_{i}\right|}}\right] .
\end{aligned}
$$

We hope to find a minimal surface of this genus. For the first case, the sign of an $n$-pretzel is $( \pm, \pm, \ldots, \pm$, even $, \mp, \mp, \ldots, \mp)$. The rule is to use the move from the outmost pair. Then the moves in Figure 6 will increase $V$ by two but will not change $E, F(=1)$; thus we get a surface with one less genus. If we represent the move by the Conway notation for algebraic links $[7]$, it is either $(\ldots,-a, \ldots, b, \ldots) \Rightarrow(\ldots,(-1)(-a$ $+1), \ldots,(b-1)(1), \ldots)$ or $(\ldots, a, \ldots,-b, \ldots) \Rightarrow(\ldots,(1)(a-1), \ldots,(-1)(-b+1), \ldots)$ where the sign sum of the $o_{i}$ 's between $a, b$ has to be zero.

For the general case, if we only look at the signs of the odd twists from $o_{1}$, we can find a pair $o_{i}, o_{j}$ such that we can apply the move we described above. The resulting diagram satisfies the same hypothesis with strictly smaller twisted bands. Inductively we get a well-defined sequence of moves which makes the desired diagram on which Seifert's algorithm will produce a minimal genus surface. Figure 6 shows the effect on $V, E$. This completes the case (i).

For the second case, $n$ is even, we can see that the degree of $\nabla_{K\left(e_{1}, o_{1}, o_{2}, \ldots, o_{n}\right)}$ is

$$
1+\operatorname{degree}\left(\nabla_{e_{1}}\right)+\prod_{i=2}^{n} \operatorname{degree}\left(\nabla_{o_{i}}\right)=\left|e_{1}\right|+\sum_{i=2}^{n}\left(\left|o_{i}\right|-1\right),
$$

and the coefficient of the leading term is $-\operatorname{sign}\left(e_{1}\right)(\alpha+\beta) / 2$ from Theorem 3.3.

Suppose $\alpha+\beta$ is nonzero. Then the Seifert surface $F$ obtained by applying Seifert's algorithm to the diagram in Figure 5 is a minimal genus surface. The genus of the Seifert surface $F_{K}$ is

$$
\begin{aligned}
g\left(F_{K}\right) & =\frac{1}{2}\left[2-\chi\left(F_{K}\right)\right]=\frac{1}{2}(2-V+E-F) \\
& =\frac{1}{2}\left[2-(n)+\left[\left|e_{1}\right|+\sum_{i=2}^{n}\left(\left|o_{i}\right|\right)\right]-1\right]=\frac{1}{2}\left[\left|e_{1}\right|+\sum_{i=2}^{n}\left(\left|o_{1}\right|-1\right)\right] \\
& =\frac{1}{2} \operatorname{degree} \nabla_{K\left(e_{1}, o_{1}, o_{2}, \ldots, o_{n}\right)} .
\end{aligned}
$$

Suppose $\alpha+\beta=0$. This means that we have the same number of positive and negative twists. As we did before we drop exactly one in the degree of the Conway 


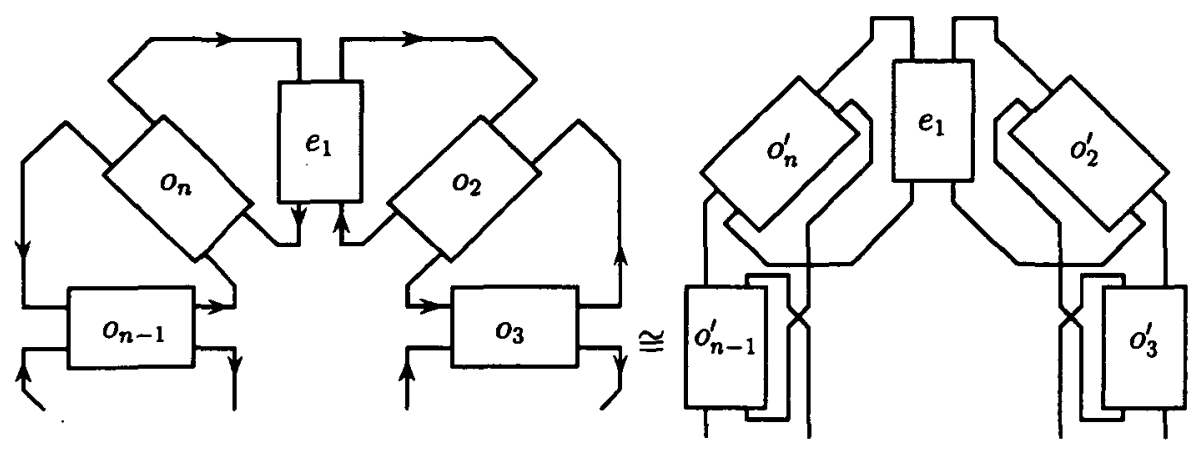

Figure 6: How to modify a diagram in Figure 5 to find a minimal genus diagram of $L\left(p_{1}, p_{2}, \ldots, p_{n}\right)$.

polynomial in equation 7 with new leading coefficient 1 . All arguments are the same if we change the term in parentheses in the equation as follows.

$$
\left[\nabla_{e_{1}^{\prime}}+\nabla_{e_{1}}\left(-\frac{\beta+\alpha}{2} z+\sum_{i=2}^{n} \frac{\nabla_{o_{i}^{\prime}}}{\nabla_{o_{i}}}\right)\right]=\nabla_{e_{1}}\left[-\frac{\beta+\alpha}{2} z+\frac{\nabla_{e_{1}^{\prime}}}{\nabla_{e_{1}}}+\sum_{i=2}^{n} \frac{\nabla_{o_{i}^{\prime}}}{\nabla_{o_{i}}}\right] .
$$

We can find a minimal surface of this genus by the same method as shown in Figure 6 if we handle the even crossing box together. This gives us the following theorem.

THEOREM 4.1. Let $K\left(p_{1}, o_{2}, o_{3}, \ldots, o_{n}\right)$ be an $n$-pretzel knot with one even $p_{1}$. Let $\alpha=\sum_{i=2}^{n} \operatorname{sign}\left(o_{i}\right)$ and $\beta=\operatorname{sign}\left(p_{1}\right)$. Suppose $\left|p_{1}\right|,\left|o_{i}\right| \geqslant 2$. Let

$$
\delta=\sum_{i=2}^{n}\left(\left|o_{i}\right|-1\right)
$$

Then the genus $g(K)$ of $K$ is

$$
g(K)= \begin{cases}\frac{1}{2}(\delta+2) & \text { if } n \text { is odd and } \alpha \neq 0 \\ \frac{1}{2} \delta & \text { if } n \text { is even and } \alpha=0, \\ \frac{1}{2}\left(\left|p_{1}\right|+\delta\right) & \text { if } n \text { is even and } \alpha+\beta \neq 0 \\ \frac{1}{2}\left(\left|p_{1}\right|+\delta\right)-1 & \text { if } n \text { is even and } \alpha+\beta=0\end{cases}
$$

4.2. GENERA OF $n$-PRETZEL LINKS. Intuitively, if we have more even $p_{i}$ 's with opposite directions, then we shall have a surface of smaller genus. So we want to choose an orientation which forces all the even $p_{i}$ 's to move in the opposite directions, but this may not be possible for all cases. 


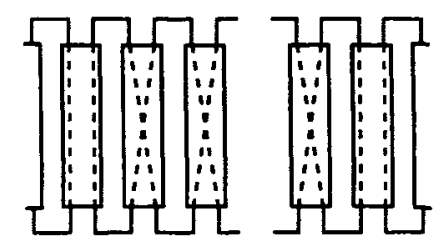

Figure 7: Boundary orientation of $L\left(p_{1}, p_{2}, \ldots, p_{n}\right)$.

Lemma 4.2. Let $L\left(p_{1}, p_{2}, \ldots, p_{n}\right)$ be an $n$-pretzel link and let $s$ be the number of even $p_{i}$ 's. If $n-s$ is even, then there exists an orientation of $L$ such that the link $L$ moves in opposite directions in all boxes of even $p_{i}$. If $n-s$ is odd and a given $p_{t}$ is even, then there exists an orientation of $L$ such that the link $L$ moves in opposite directions in all boxes of even $p_{i}$ 's except the one corresponding to $p_{t}$.

Proof: If all $p_{j}$ between two even $p_{i}$ and $p_{k}$ are odd, the number of these $p_{j}$ 's odd ( $\bmod 2$ ) will decide the boundary orientation as depicted in Figure 7.

If the number of odd crossing boxes is even, we can orient the link such that the link moves oppositely in all boxes of even crossings. Otherwise there is just one box for which the link moves in the same direction. So starting from $p_{t}$ will complete the proof.

Let us denote the orientation we choose in Lemma 4.2 by $O^{\prime}$. From Theorem 3.2, we can do almost the same comparison by using equation (1). But we have to be careful to use (1) for links. Since it was defined for oriented links, we can interpolate it as follows.

$$
\begin{gathered}
g(L)=\min _{O}\left\{\operatorname { m i n } \left\{\text { genus of } \mathcal{F}_{(L, O)} \mid \mathcal{F}_{L, O}\right.\right. \text { is a Seifert surface } \\
\text { with the orientation } O\}\} .
\end{gathered}
$$

where the first $O$ runs over all possible orientations of $L$. So (1) gives us an inequality on the second minimum of the fixed orientation $O$ and $\nabla_{(L, O)}$.

We divide into two cases:

(i) $n-s$ is even,

(ii) $n-s$ is odd.

For the first case, $n-s$ even, we can see that the degree of $\nabla_{L\left(p_{1}, p_{2}, \ldots, p_{n}\right)}$ is

$$
s+\prod_{i=1}^{n-s} \operatorname{degree}\left(\nabla_{p_{m_{i}}}\right)+1=s+\sum_{i=1}^{n-s}\left(\left|p_{m_{i}}\right|-1\right)+1,
$$

and the coefficient of this leading term is $-\alpha / 2$ from Theorem 3.2.

Suppose $\alpha$ is nonzero. Then the Seifert surface $\mathcal{F}$ obtained by applying Seifert's algorithm with the fixed orientation $O^{\prime}$ is a minimal genus surface of $\left(L, O^{\prime}\right)$. Let us find 
the genus of the Seifert surface $\mathcal{F}_{\left(L, O^{\prime}\right)}$.

$$
\begin{aligned}
2 g\left(\mathcal{F}_{L}\right) & =2-\chi\left(\mathcal{F}_{L}\right)=2-(V-E+F) \\
& =2-(n-s)+\left(\sum_{i=1}^{n-s}\left(\left|p_{m_{j}}\right|-1\right)\right)+\left[\sum_{j=1}^{s}\left|p_{i_{j}}\right|+\sum_{i=1}^{n-s}\left(\left|p_{m_{i}}\right|\right)\right]-s \\
& =2+\sum_{i=1}^{n-s}\left(\left|p_{k_{i}}\right|-1\right)=\operatorname{degree}\left(\nabla_{L}\right)-s+1 .
\end{aligned}
$$

For the rest of the cases the arguments are parallel to the argument for knots. Next, we explain how $p_{t}$ will be chosen for the rest of the article.

REMARK 4.3. First, we look at the minimum of the absolute value of $p_{e_{i}}$ over all even crossings. If the minimum is taken by the unique $p_{e_{i}}$ or by $p_{e_{i}}$ 's of the same sign, we choose it for $p_{t}$. If there are more than two $p_{e_{i}}$ 's with different signs and the same absolute value, then we look at the value $\alpha$, the sign sum of odd crossings. If it is neither 1 nor -1 , then we pick the positive one for $p_{t}$. If $\alpha=1(-1)$, pick the negative(positive) one for $\dot{p}_{t}$.

For the second case, $n-s$ odd, we find $p_{t}$ as described the above. For the last two cases, we shall drop the genus by 1 . Denote the orientation we chose here by $O_{1}$.

LEMMA 4.4. For an arbitrary orientation $O$, degree $\nabla_{(L, O)} \geqslant \operatorname{degree} \nabla_{\left(L, O_{1}\right)}$.

Proof: If we count $t_{O}$, the number of even crossings in which the link moves in the opposite directions with respect to $O$, we can see that $t_{O} \leqslant t_{O_{1}}$. If we look at the Conway polynomial in Theorem 3.3, we have that (i) we can ignore the second term, (ii) increasing $t$ by 1 will change the degree of the second term by $-\left(\left|p_{i}\right|-2\right)$, and by hypothesis, $\left|p_{i}\right| \geqslant 2$.

THEOREM 4.5. Let $L\left(p_{1}, o_{2}, \ldots, o_{s}, e_{s+1}, \ldots, e_{n}\right)$ be an $n$-pretzel link with at least one even $p_{i}$. Let $\alpha=\sum_{i=2}^{n-s} \operatorname{sign}\left(p_{o_{i}}\right)$ and $\beta=\operatorname{sign}\left(p_{t}\right)$. Suppose $\left|o_{i}\right|,\left|e_{j}\right| \geqslant 2$. Let $p_{t}$ be the integer described in Remark 4.3. Let $l$ be the number of even $p_{i}$ 's. Let

$$
\delta=\sum_{i=2}^{n-s}\left(\left|o_{i}\right|-1\right)
$$

Then the genus $g(L)$ of $L$ is

$$
g(L)= \begin{cases}\frac{1}{2} \delta+1 & \text { if } n-s \text { is even and } \alpha \neq 0 \\ \frac{1}{2} \delta & \text { if } n-s \text { is even and } \alpha=0 \\ \frac{1}{2}\left(\left|p_{t}\right|+\delta\right) & \text { if } n-s \text { is odd and } \alpha+\beta \neq 0 \\ \frac{1}{2}\left(\left|p_{t}\right|+\delta\right)-1 & \text { if } n-s \text { is odd and } \alpha+\beta=0\end{cases}
$$


Proof: It follows from Theorem 3.2, 3.3 and Lemma 4.4.

\section{THE BASKET NUMBERS OF PRETZEL LINKS}

First let us recall a definition of the basket number. Let $A_{n} \subset \mathbb{S}^{3}$ denote an $n$-twisted unknotted annulus. A Seifert surface $\mathcal{F}$ is a basket surface if $\mathcal{F}=D_{2}$ or if $\mathcal{F}=\mathcal{F}_{0}{ }^{*} A_{n}$ which can be constructed by plumbing $A_{n}$ to a basket surface $\mathcal{F}_{0}$ along a proper arc $\alpha \subset D_{2} \subset \mathcal{F}_{0}$. A basket number of a link $L$, denoted by $b k(L)$, is the minimal number of annuli used to obtain a basket surface $\mathcal{F}$ such that $\partial \mathcal{F}=L$. For standard definitions and notations, we refer to [31]. Throughout the section, we shall assume all links are not splitable, that is, Seifert surfaces are connected. Otherwise, we can handle each connected component separately.

For the basket number and the genus of a link, there is a useful theorem.

THEOREM 5.1. ([3]) Let $L$ be a link of $l$ components. Then the basket number of $L$ is bounded by the genus and the canonical genus of $L$ as,

$$
2 g(L)+l-1 \leqslant b k(L) \leqslant 2 g_{c}(L)+l-1 .
$$

Since we have found that a minimal genus surface of a pretzel link $L$ of genus $g(L)$ can be obtained by applying Seifert algorithm on a diagram of $L$, that is, $g(L)=g_{c}(L)$, we find that the basket number of a pretzel link $L$ is $2 g(L)+l-1$, that is, $b k(L)=2 g(L)+l-1$.

TheOREM 5.2. Let $K\left(p_{1}, o_{2}, o_{3}, \ldots, o_{n}\right)$ be an $n$-pretzel knot with one even $p_{1}$. Let $\alpha=\sum_{i=2}^{n} \operatorname{sign}\left(o_{i}\right)$ and $\beta=\operatorname{sign}\left(p_{1}\right)$. Suppose $\left|p_{1}\right|,\left|o_{i}\right| \geqslant 2$. Let

$$
\delta=\sum_{i=2}^{n}\left(\left|o_{i}\right|-1\right)
$$

Then the basket number $b k(K)$ of $K$,

$$
b k(K)= \begin{cases}\delta+2 & \text { if } n \text { is odd and } \alpha \neq 0, \\ \delta & \text { if } n \text { is even and } \alpha=0 \\ \left|p_{1}\right|+\delta & \text { if } n \text { is even and } \alpha+\beta \neq 0 \\ \left|p_{1}\right|+\delta-2 & \text { if } n \text { is even and } \alpha+\beta=0\end{cases}
$$

THEOREM 5.3. Let $L\left(p_{1}, o_{2}, \ldots, o_{s}, e_{s+1}, \ldots, e_{n}\right)$ be an $n$-pretzel link with at least one even $p_{i}$. Let $\alpha=\sum_{i=2}^{n-s} \operatorname{sign}\left(p_{o_{i}}\right)$ and $\beta=\operatorname{sign}\left(p_{t}\right)$. Suppose $\left|o_{i}\right|,\left|e_{j}\right| \geqslant 2$. Let $p_{t}$ be the integer described in Remark 4.3. Let $l$ be the number of even $p_{i}$ 's. Let

$$
\delta=\sum_{i=2}^{n-s}\left(\left|o_{i}\right|-1\right)
$$


Then the basket number $b k(L)$ of $L$,

$$
b k(L)= \begin{cases}\delta+l+1 & \text { if } n-s \text { is even and } \alpha \neq 0 \\ \delta+l-1 & \text { if } n-s \text { is even and } \alpha=0 \\ \left|p_{t}\right|+\delta+l-1 & \text { if } n-s \text { is odd and } \alpha+\beta \neq 0 \\ \left|p_{t}\right|+\delta+l-3 & \text { if } n-s \text { is odd and } \alpha+\beta=0\end{cases}
$$

\section{REFERENCES}

[1] C. Adams, 'Almost alternating links', Topology Appl. 46 (1992), 151-165.

[2] C. Adams, The knot book (W.H. Freeman and Company, New York 1994).

[3] Y. Bae, D. Kim and C. Park, 'Basket, flat plumbing and flat plumbing basket numbers of links', (preprint, arXiv: GT/0607079).

[4] R. Bedient, 'Double branched covers and pretzel knots', Pacific J. Math 112 (1984), 265-272.

[5] M. Brittenham, 'Free genus one knots with large volume', Pacific J. Math. 201 (2001), 61-82.

[6] D. Cimasoni, 'A geometric construction of the Conway potential function', Comment. Math. Helv. 79 (2004), 126-146.

[7] J. Conway, 'An enumeration of knots and links, and some of their algebraic properties', in Computational problems in abstract algebra (Pergamon Press, Oxford, 1970), pp. $329-358$.

[8] R. Crowell, 'Genus of alternating link types', Ann. of Math. 69 (1959), 258-275.

[9] J. Franks and R. Williams, 'Braids and the Jones polynomials', Trans. Amer. Math. Soc. 303 (1987), 97-108.

[10] D. Gabai, 'The Murasugi sum is a natural geometric operation', in Low-Dimensional Topology (San Francisco, CA, USA, 1981) (Amer. Math. Soc., Providence, RI, 1983), pp. 131-143.

[11] D. Gabai, 'The Murasugi sum is a natural geometric operation II', in Combinatorial Methods in Topology and Algebraic Geometry (Rochester, NY, USA, 1982) (Amer. Math. Soc., Providence, RI, 1985), pp. 93-100.

[12] D. Gabai, 'Genera of the arborescent links', Mem. Amer. Math. Soc. 59 (1986), 1-98.

[13] M. Hara, 'Q-polynomial of pretzel links', Tokyo J. Math. 16 (1993), 183-190.

[14] J. Hare, 'How to construct all fibered knots and links', Topology 21 (1982), 263-280.

[15] M. Hara, Y. Nakagawa and Y. Ohyama, 'The Conway potential functions for pretzel links and Montesinos links', Kobe J. Math. 6 (1989), 1-21.

[16] C. Hayashi and M. Wada, 'Constructing links by plumbing flat annuli', J. Knot Theory Ramifications 2 (1993), 427-429.

[17] J. Hoste, M. Thistlethwaite and J. Weeks, 'The first 1701936 knots', Math. Intellegencer 20 (1998), 33-48.

[18] V. Jones, 'Hecke algebra representations of braid groups and link polynomials', Ann. of Math. 126 (1987), 335-388. 
[19] F. Hosokawa and Y. Nakanishi, 'On 3-fold irregular branched covering spaces of pretzel knots', Osaka J. Math. 23 (1986), 249-254.

[20] L. Kauffman, 'Combinatorics and knot theory', Contemp. Math. 20 (1983), 181-200.

[21] M. Kobayashi and T. Kobayashi, 'On canonical genus and free genus of knot', J. Knot Theory Ramifications 5 (1996), 77-85.

[22] R. Landvoy, 'The Jones polynomial of pretzel knots and links', Topology Appl. 83 (1998), 135-147.

[23] W. Lickorish, 'Prime knots and tangles', Trans. Amer. Math. Soc. 267 (1981), 321-332.

[24] W. Menasco, 'Closed incompressible surfaces in alternating knot and link complements', Topology 23 (1984), 37-44.

[25] Y. Moriah, 'On the free genus of knots', Proc. Amer. Math. Soc. (1987), 373-379.

[26] H. Morton, 'Fibred knots with a given Alexander polynomial', in Knots, Braids and Singularities (Plans-sur-Bex, 1982) (Enseign. Math., Geneva, 1983), pp. 205-222.

[27] K. Murasugi, 'On the genus of the alternating knot, I. II', J. Math. Soc. Japan 10 (1958), 94-105, 235-248.

[28] Y. Nakagawa, 'On the Alexander polynomials of pretzel links $L\left(p_{1}, \ldots, p_{n}\right)$ ', Kobe $J$. Math. 3 (1987), 167-177.

[29] T. Nakamura, 'On canonical genus of fibered knot', J. Knot Theory Ramifications 11 (2002), 341-352.

[30] L. Rudolph, 'Quasipositive annuli (Constructions of quasipositive knots and links IV.)', J. Knot Theory Ramifications 1 (1992), 451-466.

[31] L. Rudolph, 'Hopf plumbing, arborescent Seifert surfaces, baskets, espaliers, and homogeneous braids', Topology Appl. 116 (2001), 255-277.

[32] M. Sakuma, 'Minimal genus Seifert surfaces for special arborescent links', Osaka J. Math. 31 (1994), 861-905.

[33] H. Seifert, 'Uber das Geschlecht von Knoten', Math. Ann. 110 (1934), 571-592.

[34] Y. Shinohara, 'On the signature of pretzel links', in Topology and computer science(Atami, 1986) (Kinokuniya, Tokyo, 1987), pp. 217-224.

[35] J. Stallings, 'Constructions of fibred knots and links', in Algebraic and Geometric Topology, (Proc. Sympos. Pure Math., Stanford Univ., Stanford, CA, 1976), Part 2 (Amer. Math. Soc., Providence, RI, 1978), pp. 55-60.

[36] G. Torres, 'On the Alexander polynomail', Ann. of Math. 57 (1953), 57-89.

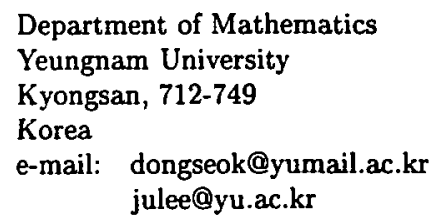

